Pensamiento Crítico N. ${ }^{\circ}$ 13, pp. 91-99

\title{
Diamond, Mortensen y Pissarides: Premios Nobel de Economía 2010 e implicancias para la economía peruana
}

\author{
Richard Roca ${ }^{1}$ \\ rhroca@yahoo.com
}

\section{RESUMEN}

El presente artículo presenta un resumen de los aportes de los nuevos Premios Nobel de Economía, los profesores Diamond, Mortensen y Pissarides y sus principales publicaciones. Además, se presenta una reflexión sobre las principales implicancias para la economía peruana.

Palabras clave: Premio Nobel de Economía, mercado laboral, mercados imperfectos, búsqueda, descalce.

\section{ABSTRACT}

This article presents a summary of the contributions of the new Nobel Prizes in Economics, the professors Diamond, Mortensen and Pissarides and their main publications. In addition it presents a reflection on the principal implications for the Peruvian economy

Keywords: Nobel Prize of Economy, labor market, imperfect markets, search, mismatching.

1 Profesor principal de Economía de la Universidad de San Marcos, Pontificia Universidad Católica del Perú y Ricardo Palma. Magíster en Economía por la Pontificia Universidad Católica del Perú y candidato a Doctor en la misma especialidad. Página web: http://richardroca.blogspot.com 


\section{Los galardonados y sus aportes}

El Banco Central de Suecia (Sveriges Riksbanks), fundado en 1668, considerado como el Banco Central más antiguo del mundo, anunció que el "Premio en Ciencias Económicas del Banco de Suecia en honor de Alfred Nobel" del 2010, conocido popularmente como "Premio Nobel de Economía", ha sido otorgado a los profesores estadounidenses Peter A. Diamond, profesor del famoso Instituto Tecnológico de Massachusetts (MIT), Dale T. Mortensen, profesor de la Northwestern University, y al greco-chipriota nacionalizado británico Christopher A. Pissarides, profesor de la London School of Economics por sus aportes al "Análisis de mercados con imperfecciones en los procesos de búsqueda", con modelos matemáticos que describen las dificultades que enfrentan los compradores y vendedores para entrar en contacto, las dificultades de acoplamiento de la oferta y la demanda de los mercados reales. La Academia Sueca destaca, entre otras cosas, que las investigaciones de los nuevos Premios Nobel explican por qué la gente sigue desempleada aun cuando hay muchas vacantes. Además, ayuda a comprender cómo el desempleo, las vacantes y los salarios son afectados por la regulación y la política económica.
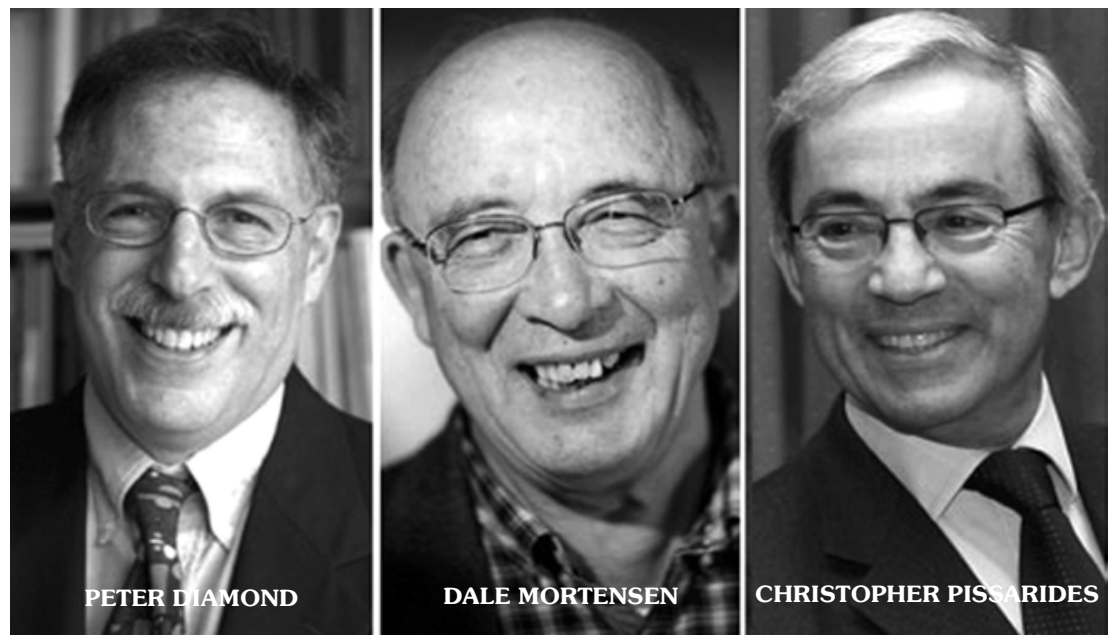

Sus estudios analizan casos como los del mercado de trabajo en los cuales se puede observar que habiendo vacantes de trabajo por cubrir y trabajadores que pueden entrar a dichos puestos, sin embargo están desempleados. Los trabajadores tratan de 


\section{Richard Roca}

encontrar el mejor empleo mientras que las empresas tratan de contratar los mejores trabajadores. Consideran los problemas de los costos de búsqueda en contexto de información imperfecta, cuanto mayor sean los costos o dificultades de búsquedas mayor el nivel y el tiempo del desempleo.

Además, consideran que los beneficios sociales de desempleo muy generosos alargan las temporadas de alto desempleo. Los trabajadores pasan más tiempo buscando mejores empleos, por lo tanto, permaneciendo más tiempo desempleados. Su teoría de búsqueda ha sido aplicada a otros fenómenos como los mercados de vivienda, la demanda de dinero, el caso de los divorcios y otros temas.

Peter Diamond, de 70 años, se graduó de bachiller en matemáticas en 1960 en la universidad de Yale y obtuvo el grado de doctor en economía (PhD) en 1963 en el Massachussetts Institute of Technology. Actualmente es Institute Professor en el MIT. De Diamond se destacan sus "contribuciones decisivas a la teoría de mercados de búsqueda y emparejamiento", Adicionalmente ha hecho valiosos aportes al estudio de los seguros sociales, fondos de pensiones y de las finanzas públicas en un contexto intertemporal y el crecimiento económico. El profesor Diamond ha señalado recientemente que EE.UU. necesita de un nuevo impulso fiscal para evitar caer en una nueva recesión. Señalo que el programa aplicado por el gobierno de Obama y la FED eran necesarios, que sin esas medidas la crisis actualmente sería mucho más graves, el desempleo sería mucho mayor, pero que requiere un impulso adicional para salir más rápido de dicha crisis. Curiosamente a principios de este año Diamond había sido propuesto por Obama para ocupar un cargo en la FED y su propuesta fue objetada por el Senador Republicano Richard Shelby por considerar que no estaba suficientemente calificado. Es muy probable que se reconsidere su caso.

Dale Mortensten, de 71 años, se graduó en Economía en 1961 por la Willamette University y en 1967 obtuvo su doctorado por la Carnegie-Mellon University. Ha sido profesor en las Universidades Carnegie-Mellon y Northwestern. Mortensen es conocido por su trabajo pionero en la búsqueda y la teoría del juego de desempleo friccional.

Mortensen encontró que la tasa de salida del desempleo aumenta cuando se acerca el final de las prestaciones. Aunque cierto nivel de prestaciones puede no solo aumentar el bienestar sino también contribuir a un mejor funcionamiento del mercado de trabajo, 


\section{Pensamiento Crítico N. 13}

pues permite prolongar la búsqueda de empleo hasta lograr el resultado más eficiente. Esos descubrimientos ayudarían a plantear diseños alternativos de las prestaciones por desempleo.

Pissarides, de 62 años, obtuvo el bachillerato en Economía en 1971 en la Universidad de Essex y el doctorado en economía en 1973 en la London School of Economics en la cual actualmente es profesor y director del Programa de Investigación sobre Macroeconomía en el Centro para el Desempeño Económico.

Mortensen y Pissarides profundizaron las investigaciones de Diamond y analizaron el problema del desempleo en varios países. Entre ambos publicaron una serie de trabajos una de las cuales, la de 1994, titulada: "Job Creation and Job Destruction in the Theory of Unemployment", considerada como una de las mejores, analiza la persistencia del desempleo y los shocks negativos que aumentan la inestabilidad laboral dada la volatilidad que tiene la creación de empleo.

Según la Academia sueca, el modelo Diamond-Mortensen-Pissarides DMP es la "herramienta más usada" para analizar el problema del desempleo, cómo las compañías reclutan trabajadores y sobre como se forman los salarios.

El "Premio Nobel de Economía" fue creado en 1969 y financiado por el Banco Central de Suecia, el premio es designado por un comité y consta de una dotación de 10 millones de coronas suecas (aproximadamente 1,44 millones de dólares ó 1,08 millones de euros). Es equivalente al monto otorgado en los otros premios de la Fundación Nobel.

En setiembre de cada año el Comité Nobel de Economía, que consiste en cinco miembros elegidos, envía invitaciones a cientos de científicos, miembros de academias y profesores de universidad en númerosos países, pidiéndolos sugerencias para denominar a candidatos por el Premio en la Economía para el año siguiente. También consultan a los miembros de la Academia y antiguos laureados para denominar a candidatos. Todas las propuestas deben ser recibidas antes del 1 de febrero. Las propuestas son evaluadas por el Comité. Antes del finalizar el mes de setiembre, el comité escoge a los potenciales ganadores. Si hay un empate, el presidente del comité decide. Después, los potenciales laureados deben ser aprobados por la Academia Real Sueca de Ciencias. En octubre votan para determinar al ganador o ganadores del Premio Nobel de Economía. 


\section{Richard Roca}

Como en los demás premios Nobel, no más que tres personas pueden compartir el premio de un año dado, deben estar vivos al momento del anuncio. Reciben un diploma, una medalla de oro y el documento de premio de subvención monetario de manos del Rey de Suecia en la Ceremonia de Premio Nobel anual en Estocolmo, el 10 de diciembre - el aniversario de la muerte de Nobel. La información sobre las nominaciones de las candidaturas no puede ser revelada hasta dentro de 50 años.

\section{Implicancias para el mercado laboral peruano}

El mercado laboral peruano se caracteriza por tener no solo elevados niveles de desempleo sino también altas tasas de subempleo, fenómeno casi desconocido en los países desarrollados.

En buena medida el desempleo se debe a la insuficiencia de la inversión, pero también a que existen serios problemas de información. Muchas empresas no saben dónde y cómo ubicar a los trabajadores que necesitan contratar, mientras que muchísimos trabajadores no saben que empresas los podrían contratar dando lugar a una mayor tasa desempleo.

Para reducir estos problemas de información el Gobierno mediante el Ministerio de Trabajo podría publicar una página web en la que todas las empresas privadas y públicas, anuncien obligatoriamente sus demandas de trabajado, por área económica u región económica, en la cual los que buscan trabajo hallarían los puestos de trabajo que más les conviene.

Así mismo, el gobierno podría orientar a los trabajadores y jóvenes estudiantes acerca de qué carreras técnicas y profesionales seguir de acuerdo a estudios continuos que el Ministerio de Trabajo tendría que hacer sobre la demanda y oferta laboral de los diversos sectores y de la economía

Adicionalmente, el Gobierno debería promover y priorizar la formación laboral en aquellas especialidades en las que haya mejores perspectivas, tanto para los trabajadores como para las empresas, elevando no solo el nivel del empleo sino también las remuneraciones al evitar la sobre oferta de determinadas profesiones como sucede en la actualidad. Todo esto tiene que ver con el sistema educativo de nuestro país el 


\section{Pensamiento Crítico N. 13}

cual requiere de cambios sustanciales mejorando no solo la calidad de la enseñanza sino también de una reorientación radical, sobre todo la educación secundaria, la cual debería de capacitar a los estudiantes en alguna carrera técnica que tengan alta demanda de tal forma que al terminar la secundaria, ya estén aptos para conseguir un trabajo, no solo más productivo sino también mejor remunerado.

\section{Principales investigaciones de los nuevos Premios Nobel de Economía del 2010}

Entre las principales publicaciones de los nuevos galardonados se puede mencionar los siguientes:

Blanchard, O. y P., Diamond (1990). The aggregate matching function. En P diamond (ed.), Growth, Productivity, Unemployment, MIT Press.

Burdett, K. y D., Mortensen (1998). "Wage differentials, employer size and unemployment". En: International Economic Review.

Diamond, P. (1965). "National debt in a neoclassical growth model". En: American Economic Review.

Diamond, P. (1967). "The role of a stock market in a general equilibrium model with technological uncertainty". En: American Economic Review.

Diamond, P. (1971). "A model of price adjustment". En: Journal of Economic Theory.

Diamond, P. (1981). "Mobility costs, frictional unemployment, and efficiency". En: Journal of Political Economy.

Diamond, P. (1982a). "Wage determination and efficiency in search equilibrium". En: Review of Economic Studies.

Diamond, P. (1982b). "Aggregate demand management in search equilibrium". En: Journal of Political Economy. 


\section{Richard Roca}

Diamond, P. (1984). A search-equilibrium approach to the micro foundations of macroeconomics. MIT Press.

Diamond, P. y D. Fudenberg (1989). "Rational expectations business cycles in search equilibrium". En: Journal of Political Economy.

Diamond, P. y E. Maskin (1979). "An equilibrium analysis of search and breach of contract, I: steady states". En: Bell Journal of Economics.

Diamond, P. y E. Maskin (1981). "An equilibrium analysis of search and breach of contract, II: a non-steady state example". En: Journal of Economic Theory.

Diamond, P. y J. Mirrlees (1971a). "Optimal taxation and public production I: production efficiency". En: American Economic Review.

Diamond, P. y J. Mirrlees (1971b). "Optimal taxation and public production II: tax rules". En: American Economic Review.

Diamond, P. y J. Mirrlees (1978). "A model of social insurance with variable retirement". En: Journal of Public Economics.

Mortensen, D. (1970a). "A theory of wage and employment dynamics". En: E. S. Phelps et al., Microeconomic Foundations of Employment and Inflation Theory, Macmillan.

Mortensen, D. (1970b). "Job search, the duration of unemployment and the Phillips curve". En: American Economic Review.

Mortensen, D. (1977). "Unemployment insurance and job search decisions". En: Industrial and Labor Relations Review.

Mortensen, D. (1978). "Specific capital and labor turnover". En: Bell Journal of Economics. 


\section{Pensamiento Crítico N. 13}

Mortensen, D. (1982a). "The matching process as a noncooperative bargaining game". En: J McCall (ed.), The Economics of Information and Uncertainty, University of Chicago Press.

Mortensen, D. (1982b). "Property rights and efficiency in mating, racing and related games". En. American Economic Review.

Mortensen, D. (1988). "Matching: finding a partner for life or otherwise". En: American Journal of Sociology (Supplement).

Mortensen, D. (1994a). "The cyclical behavior of job and worker flows". En: Journal of Economic Dynamics and Control.

Mortensen, D. (1994b). "Reducing supply-side disincentives to job creation". En: Reducing Unemployment: Current Issues and Policy Options, Federal Reserve Bank of Kansas City.

Mortensen, D. (2005). Wage dispersion: why are similar workers paid differently? MIT Press.

Mortensen, D. y C. Pissarides (1994). "Job creation and job destruction in the theory of unemployment". En: Review of Economic Studies.

Mortensen, D. y C. Pissarides (1998). "Technologial progress, job creation and job destruction". En: Review of Economic Dynamics.

Mortensen, D. y C. Pissarides (1999a). "Unemployment responses to 'skillbiased' technology shocks: the role of labour market policy". En: Economic Journal.

Mortensen, D. y C. Pissarides (1999b). "New developments in models of search in the labor market". En: O Ashenfelter and D Card (eds.), Handbook of Labor Economics, vol. 3, part. 2, Elsevier.

Mortensen, D. y C. Pissarides (1999c). "Job reallocation, employment fluctuations and unemployment". En: J Taylor y M. Woodford (eds.), Handbook of Macroeconomics vol. 1, part. 2, Elsevier. 


\section{Richard Roca}

Mortensen, D. y E. Nagypal (2007). "More on unemployment and vacancy fluctuations". En: Review of Economic Dynamics.

Petrongolo, B. y C. Pissarides (2001). "Looking into the black box: a survey of the matching function". En: Journal of Economic Literature.

Pissarides, C. (1979). "Job matching with state employment agencies and random search". En: Economic Journal.

Pissarides, C. (1984a). "Search intensity, job advertising, and efficiency". En: Journal of Labor Economics.

Pissarides, C. (1984b). "Efficient job rejection". En: Economic Journal 94 (Supplement).

Pissarides, C. (1985). "Short-run equilibrium dynamics of unemployment, vacancies, and real wages". En: American Economic Reivew.

Pissarides, C. (1990/2000). Equilibrium unemployment theory, Basil Blackwell; second edition, MIT Press.

Pissarides, C. (1992). "Loss of skill during unemployment and the persistence of employment shocks". Quarterly Journal of Economics.

Pissarides, C. (1994). "Search unemployment with on-the-job search". En: Review of Economic Studies.

Pissarides, C. (2009). "The unemployment volatility puzzle: is wage stickiness the answer?" En: Econométrica. 